Project 1011901

\title{
Ecological Interactions Between Metals and Microbes
}

Konopka, Allan E.

Purdue University

\begin{abstract}
RESULTS TO DATE: 1 . Analysis of Lead Resistant Arthrobacter sp. SI-1 Arthrobacter sp. SI-1 was isolated from contaminated soils at the Seymour site, and was found to be resistant to $\mathrm{Pb}$ at concentrations near its solubility limit (150 micromolar). The genetic region that confers lead resistance is located on a plasmid (PSI-1)has been cloned. We have continued to analyze the sub-clones from the pSI1 region. Initially we had predicted that ORF1-ORF5 were involved in lead resistance because their organization suggest a potential operon. In addition these same five genes have been found in a similar organization on a plasmid from Arthrobacter FB24, while the pAA1 plasmid from A. aurescens TC1 contains three of the five genes. In order to determine the minimum genes required for lead resistance a series of deletion mutants were constructed from the $14.7 \mathrm{~kb}$ clone pKJ60. Deletion of ORFs 3-5 did not have any measurable effect on the ability of the cloned fragment to rescue the lead resistance phenotype in a lead sensitive strain of E. coli (RW3110). The construct pKJ65 was generated by removing approximately $200 \mathrm{bp}$ from the center region of ORF2, which codes for the P-Type ATPase; as expected this deletion resulted in a lead sensitive phenotype. While the genes downstream of ORF 2 do not appear to play a significant role in lead resistance the same cannot be said for ORF1which is upstream. Based on amino acid sequence homology a BLAST search indicates ORF1 is likely a regulatory protein from the ArsR family. When ORF1 is removed (pKJ64, pKJ67), a lead sensitive phenotype occurs. Approximately
\end{abstract} $100 \mathrm{bp}$ from the sequence of ORF1 was deleted (pKJ70) in order to test if ORF1 is required for lead resistance, or if the cells require something in the upstream non-coding region (binding site, promoter). Cells with pKJ70 show some limited growth in the presence lead, but it is generally much slower than the lead resistant constructs where ORF1 is present. These results suggest that ORF1 has a positive effect on lead resistance, perhaps acting as an activator of transcription. We are currently working to repeat this same set of experiments using cadmium. Previous work on the physiology of lead resistance was done in a MES buffered minimal media at pH6.5, the concentration of $\mathrm{PbNO} 3$ in these experiments ranged from 0 to 200 micromolar. In an effort to increase the solubility and therefore the bio-availability of the lead the $\mathrm{pH}$ was lowered to 6.1. The most significant result was the effect of the lead on the cultures at high concentrations (150 to 200 micromolar). At pH 6.5 no dose dependent response is observed with the wild type cells (SI-1, SI-2); however at $\mathrm{pH} 6.1$ the growth rate and yield is decreased for $\mathrm{SI}-1$ in the presence of 200 micromolar Pb while SI-2 showed decreases in the presence of 150 and 200 micromolar $\mathrm{Pb}$. As mentioned above ORF1 most closely matches the ArsR family of regulatory proteins which typically act as repressors, blocking transcription in the absence of metals. Activators have been shown to regulate metal resistance, however these proteins are typically MerR type regulators. We are currently working to design experiments to test the regulatory function of the ORF1 protein. Potential experimental approaches include Real Time Reverse Transcriptase PCR, Reporter Gene Assays (?-galactosidase, GFP), and biochemical assays such as band shift and nuclease protection. ORF1 has been cloned into a protein expression vector, and we have previously been able to over-express and partially purify it. The availability of sequence data from two genome sequencing projects (FB24 and TC1) along with published (pAO1) and unpublished (pSI1, pCR15) enables us to use a comparative genomics approach to look at and analyze the different functions located on these plasmids. The genes required for plasmid survival,( i.e., replication, conjugation and partitioning) are of particular interest since little is known about these functions in Arthrobacter. A group of putative conjugation genes were identified on pSI-1 (ORFs 20 to 32), while some of the genes in this group share homology to known conjugation genes (traG) many are hypothetical proteins. A comparison of pSI-1 to other Arthrobacter plasmids shows that the complete set of genes are found on the plasmids pAO1 and pFB24. In addition to sharing sequence homology the genes are largely found in the same order and orientation as on pSI1. Two sets of the conjugation genes are present on pFB24, cluster one is intact while the second cluster has approximately $6 \mathrm{~kb}$ inserted between the genes. The second set of genes may have resulted from the co-integration between pFB24 and another plasmid or conjugative transposon. While pAA1 has some of these same genes, the entire group is not present, however pAA1 carries genes related to conjugation not found on pSI-1 and may 
therefore have similar but not identical mechanism for conjugation. Predictive programs exist for identification of the origin of replication of plasmids, however they are limited to theta replicating plasmids with a single origin. We have been working with two programs ORILOC and ORIX, both operate on the principle of GC skew; however they differ in the algorithm used to calculate the cumulative skew. Preliminary results suggest that these programs, particularly ORILOC, may be able to find the origin. Based on the current dataset ORILOC predicts the origin to be in the intergenic space near the parA/soj genes, which is consistent with other experimentally tested origins or replication. However the confidence in the prediction is low because we are using raw data from GLIMMER (predicts ORFs) rather than refined data. All of the plasmids examined share genes for homologs of the par and soj family of genes, which are involved in the partioning of large low-copy number plasmids. Typically these genes are found adjacent to one another (i.e., parAB). However in pSI-1 these two genes are separated by approximately $50 \mathrm{~kb}$. Further analysis of the remaining plasmids is needed to identify additional partioning genes that may not share homology to those on pSI-1.

Proteomic analysis of chromium resistance Chromium resistant Arthrobacter sp. FB24 was previously isolated from the sampling site at Seymour, IN. This strain is resistant to $200 \mathrm{mM} \mathrm{Cr}$. The inducibility of $\mathrm{Cr}$ resistance was tested in $0.2 X$ nutrient broth, by amending cultures with either $5 \mathrm{mM}$ or $20 \mathrm{mM}$ chromate in early log phase. No lag phase was seen following the addition of $\mathrm{Cr}$, but the growth rate and yield decreased in the $\mathrm{Cr}$-stressed cultures. This may indicate that $\mathrm{Cr}$ resistance in FB24 was due to a constitutively expressed, energy demanding mechanism. The genome of strain FB24 has recently been sequenced. An analysis of the genome sequence revealed a cluster of putative chromate resistance genes located on an apparent $230 \mathrm{~kb}$ megaplasmid. Among the predicted ORFs is ChrB $(40 \%$ similarity to ChrB, Desulfovibrio vulgaris), an ORF that is $34 \%$ similar to a probable chromate resistance signal peptide protein (Ralstonia solanacearum), and two chromate ion transporters. The first putative transporter exhibits 51\% similarity to ChrA (Rhizobium meliloti), while the second is $53 \%$ similar to ChrA (Wautersia eutropha). ChrA has been shown to function in chromate efflux in other bacteria, whereas $\mathrm{ChrB}$ is thought to serve a regulatory role. A focused DNA microarray was conducted to evaluate the expression of these genes and the other putative ORFs within this gene cluster as a function of $\mathrm{Cr}$ concentration. A dose-response for chrA and chrB was seen. A malate/quinone oxidoreductase, a probable conserved lipoprotein and an uncharacterized protein also increased expression as a function of $\mathrm{Cr}$ concentration. These results suggest that one mechanism of $\mathrm{Cr}$ resistance in Arthrobacter sp. FB24 is efflux, with possible involvement of $\mathrm{Cr}$ reduction. The gene expressing the uncharacterized protein was one of the more highly expressed genes in the array and may represent a novel gene involved in $\mathrm{Cr}$ resistance. These genes are being cloned for expression in a $\mathrm{Cr}$ sensitive Arthrobacter host. Following the recovery of a $\mathrm{Cr}$ resistant phenotype, mutational analysis will be conducted to determine the extent to which each gene contributes to $\mathrm{Cr}$ resistance. Two-dimensional protein gel electrophoresis was used to examine the $\mathrm{Cr}$ response of strain FB24 on a global scale. Whole cell protein extracts were made from mid-log cells grown in $0 \mathrm{mM}, 5 \mathrm{mM}$ and $20 \mathrm{mM} \mathrm{Cr}$, added as $\mathrm{K} 2 \mathrm{CrO} 4$. Cell pellets were disrupted by sonication in a solution containing Nonident P-40, urea, 2-mercaptoethanol and 8-10 ampholytes. 2D gels and analyses were performed at Argonne National Laboratory. Two methods of separation were utilized in the first dimension to separate proteins according to their isoelectric points. Isoelectric focusing was conducted to separate proteins with pls primarily between 4-7. Nonequilibrium pH Gradient Gel Electrophoresis (NEPHGE) was used to separate basic proteins with pls above 7. Standard techniques for SDS-PAGE were used to separate proteins in the second dimension according to molecular weight in a linear gradient of $10-17 \%$ acrylamide. All gels were silver-stained and scanned on an Eikonix 1412 CCD scanner interfaced with a VAXstation 9000-40. To detect differentially expressed proteins in the pl 4-7 range, three replicate gels from each test condition were chosen for analysis using Progenesis software. Statistically significant protein spots were determined using student?s t-test, comparing the $5 \mathrm{mM}$ and $20 \mathrm{mM} \mathrm{Cr}$ gels to the $\mathrm{OmM} \mathrm{Cr}$ gels. A probability cut off of 0.05 was used. Only those proteins that differed in expression by a factor greater than 2.0 or less than 0.6 were considered in the analysis. The data revealed up-regulation of 5 proteins and down-regulation of 6 proteins with the $5 \mathrm{mM} \mathrm{Cr}$ samples; whereas 9 proteins were up-regulated and 10 were down-regulated in $20 \mathrm{mM} \mathrm{Cr}$. Of these, four proteins were differentially expressed in both $\mathrm{Cr}$ concentrations. The results of this experiment will be validated with a greater sample size. In the meantime, eight spots were cut from 6-9 replicate gels for identification by mass spectrometry (MS). The peptide map fingerprints (PMF) derived from MS will be compared to a theoretical PMF of each protein-coding region within the FB24 genome. In this manner, the gene 
encoding the differentially expressed proteins can be directly identified. Those genes of particular interest (i.e. novel or not previously associated with $\mathrm{Cr}$ resistance) will be subjected to targeted mutagenesis to verify their role in $\mathrm{Cr}$ resistance. The NEPHGE gels were compared by eye. A large number of lowmolecular weight $(10-20 \mathrm{kDa})$, highly basic proteins spots were present on the gels. At least two of these spots were either absent or less dense on the $5 \mathrm{mM}$ and $20 \mathrm{mM}$ Cr gels compared to the $0 \mathrm{mM}$ controls. Proteins that fall within this size and pl range are likely to be nucleic acid-binding proteins such as ribosomal protein subunits. Differences were seen in other regions of the gels as well. However, the evaluation was hindered by inconsistencies in the protein separation among replicate gels. These gels will be repeated to achieve more consistent replicate profiles in order to perform more reliable gel comparisons.

DELIVERABLES: Shi, W., Bischoff, M., Turco, R.F. and Konopka, A. 2005. Microbial catabolic diversity in soils contaminated with hydrocarbons and heavy metals. Environm Sci Technol 39: 1974-1979.

Becker, J.M., Parkin, T., Nakatsu, C.H., Wilbur, J.D., and Konopka, A. 2005. Bacterial activity, community structure, and cm-scale spatial heterogeneity in contaminated soil. Microb. Ecol., in press.

Joynt, J. Bischoff, M., Turco, R., Konopka, A. and Nakatsu, C.H. 2005. Microbial community analysis of soils contaminated with lead, chromium and petroleum hydrocarbons. Microb. Ecol., in press.

Nakatsu, C.H., Carmosini, N., Baldwin, B., Beasley, F., Kourtev, P. and Konopka, A. 2005. Soil microbial community responses to additions of organic carbon substrates and heavy metals ( $\mathrm{Pb}$ and $\mathrm{Cr}$ ). Appl. Environm. Microbiol., in press.

Kourtev, P. S., C. H. Nakatsu, and Konopka, A. 2006. Anaerobic bacterial community responses to organic $\mathrm{C}$ addition in chromium (VI) and iron (III) amended microcosms. Applied And Environmental Microbiology, in press. 